Recepción: 20 / 04 / 2017

Aceptación: 20 / 05 / 2017

Publicación: 15 / 07 / 2017

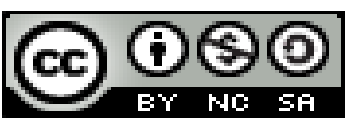

Ciencias de la Educación

Artículo Científico

\title{
Incidencia de las estrategias metodológicas en la formación académica
}

\section{Incidence of methodological strategies in academic training}

\section{Incidência de estratégias metodológicas em educação}

\author{
Mónica R. Ortiz-David ${ }^{\mathrm{I}}$ \\ monikortiz21@hotmail.com \\ Marjorie del Rocío Loor-Aldás II \\ marjoloorr@gmail.com \\ Rosa C. Varas-Giler III \\ rosac.varas@gmail.com \\ Rosa M. Chicaiza-Chicaiza IV \\ rosamchica@gmail.com
}

Correspondencia: monikortiz21@ hotmail.com 


\section{Resumen}

En la presente investigación se realiza un diagnóstico y revisión de las metodologías utilizadas en la enseñanza de la asignatura de Inglés con el objetivo de analizar la incidencia de las estrategias metodológicas en la formación académica de los estudiantes del cuarto semestre en la asignatura de Inglés de la Facultad de Psicología de la Universidad de Guayaquil para, con los resultados de este estudio, diseñar una guía de estrategias metodológicas. La investigación se llevó a cabo a través de un estudio descriptivo y analítico, mediante encuestas realizadas a Docentes de la misma área académica y a los estudiantes de la asignatura de inglés de la Facultad. Se realizó por medio de una investigación de campo. Este proyecto aportará antecedentes que si bien corresponden a una realidad particular, puede aplicarse a diferentes Universidades del país, además de aportar a conocer el rendimiento de los alumnos universitarios. En esta investigación se reconoce la importancia y necesidad de revisar dichas estrategias metodológicas para lograr así que los alumnos se sientan altamente motivados y comprometidos con el aprendizaje, permitiendo así que sean capaces de asumir su responsabilidad con claro conocimiento de su misión, como es el de mejorar su rendimiento académico durante y al final de sus estudios.

Palabras claves: Estrategias; aprendizaje; formación; académica; metodología. 


\section{Summary}

In the present investigation a diagnosis and revision of the methodologies used in the teaching of the subject of English with the objective of analyzing the incidence of the methodological strategies in the academic formation of the students of the fourth semester in the subject of English of the Faculty of Psychology, University of Guayaquil, with the results of this study, to design a guide of methodological strategies. The research was carried out through a descriptive and analytical study, by means of surveys carried out on teachers of the same academic area and students of the English course of the Faculty. It was done by means of a field investigation. This project will provide background information that, although corresponding to a particular reality, can be applied to different universities in the country, as well as contributing to the performance of university students. This research recognizes the importance and necessity of reviewing these methodological strategies in order to make students feel highly motivated and committed to learning, allowing them to be able to assume their responsibility with clear knowledge of their mission, Improve their academic performance during and at the end of their studies.

Key words: Strategies; learning; training; academic; methodology. 


\section{Resumo}

Na presente investigação a avaliação e revisão das metodologias utilizadas no ensino de Inglês como um assunto a fim de analisar o impacto das estratégias metodológicas na formação acadêmica dos alunos do quarto semestre na disciplina de Inglês, é realizada Faculdade de Psicologia da Universidade de Guayaquil, com os resultados deste estudo, concepção de um metodológicos estratégias de guia. A pesquisa foi realizada através de um estudo descritivo e analítico por meio de pesquisas de professores dos mesmos acadêmicos e estudantes de Inglês como um assunto da Faculdade. Ele foi conduzido através de pesquisa de campo. Este projecto irá fornecer fundo que, embora correspondam a uma realidade particular, pode ser aplicado a diferentes universidades do país, além de contribuir para compreender o desempenho de estudantes universitários. Nesta pesquisa a importância ea necessidade de reexaminar estas estratégias metodológicas para conseguir que os alunos se sentem muito motivados e comprometidos com a aprendizagem é reconhecida, permitindo que eles sejam capazes de assumir a sua responsabilidade com a compreensão clara da sua missão, como é o melhorar o seu desempenho acadêmico durante e no final de seus estudos.

Palavras-chave: Estratégias; aprendizagem; formação; academic; metodologia. 


\section{Introducción.}

Aprender el idioma inglés es de gran importancia porque se ha convertido en una herramienta indispensable en el mundo profesional, ya que se lo puede emplear en diversas áreas y además ayuda a romper las barreras de comunicación entre las personas, ayudando así a crecer personal y profesionalmente.

Anteriormente solo se le exigía a los altos cargos de las empresas saber inglés, pero hoy en día es un requisito en casi todos los trabajos. Estudios muestran el desinterés de los estudiantes universitarios por aprender el idioma inglés, lo que ha dado como resultado el bajo rendimiento académico de los mismos, debido al incumplimiento de las tareas y obligaciones curriculares.

Los docentes de inglés deben incentivar a los estudiantes a aumentar su interés por el idioma, ya que no solo lograrán que terminen sus estudios con buenas calificaciones, sino permitirá que estén capacitados profesionalmente para un mundo laboral cada día más competitivo.

\section{Materiales y métodos.}

Al realizar la investigación y determinar con claridad las causas del problema se considera que este proyecto es de carácter factible ya que su formulación y ejecución se apoyara en investigaciones de tipo documental por las investigaciones realizadas en libros, folletos, internet, entre otros.

El trabajo de investigación también se enmarca en la modalidad de un análisis de campo que consistió en la obtención de datos en relación directa del investigador con la realidad sin controlar o manipular variable alguna, porque se realizó la observación y constató el problema y el medio en que se desenvuelve además porque el proceso investigativo permitió el conocimiento más a fondo del investigador y pudo manejar los datos con más seguridad y podrá soportarse en diseños 
descriptivos, para dar solución al problema planteado con el tema: Incidencia de las estrategias metodológicas en la formación académica de los estudiantes del cuarto semestre en la asignatura de Inglés de la facultad de Psicología de la Universidad de Guayaquil, en el año 2015. Propuesta: Diseño de una guía de estrategias metodológicas.

\section{Tipos de Investigación.}

- Investigación bibliográfica: Al realizar la investigación se utilizó documentos impresos y virtuales, folletos y textos para obtener la información necesaria para la solución del problema.

- Investigación documental: Para la realización del proyecto se analizó todo tipo de documentos para obtener datos con información amplia y precisa.

- Investigación Básica: Mediante la aplicación de la propuesta del diseño de una guía metodológica, el docente contará con una herramienta indispensable que desarrollará competencias y habilidades cognitivas y procedimentales de los estudiantes de educación superior que les ayudará a participar activamente en su entorno educativo y social.

- Investigación de campo o Mixta: Se toma como criterio el lugar y los recursos donde se obtiene la información requerida para dar solución al problema planteado.

- Investigación descriptiva: Los investigadores recogen los datos sobre la base de una hipótesis o teoría, exponen y resumen la información de manera cuidadosa y luego analizan minuciosamente los resultados, a fin de extraer generalizaciones significativas que contribuyan al conocimiento.

\section{Población y Muestra}

El tamaño de la población es finita y corresponde a los estudiantes del cuarto semestre en la asignatura de inglés de la Facultad de Psicología de la Universidad de Guayaquil, en el año 2015. 
Se utilizó como muestra 90 estudiantes, 6 docentes y 3 autoridades para un total de 99 personas.

\section{Instrumentos de Investigación}

- Entrevista

- Encuesta

- Observación

\section{Resultados.}

- Sólo el $31.11 \%$ de los estudiante creen que los docentes aplican estrategias metodológicas que desarrollan habilidades cognitivas, sin embargo a pesar de que, según el análisis de resultados, muy pocos docentes no aplican estrategias, el $71.77 \%$ de ellos están de acuerdo en brindar el apoyo necesario para la aplicación de la guía de Estrategias Metodológicas presentadas en esta investigación. Estos dos resultados resaltan claramente los resultados de la investigación y muestran el apoyo que los estudiantes pueden brindar al cumplir con la guía presentada.

- El $71.11 \%$ de los alumnos consideran que las estrategias metodológicas beneficiará a desarrollar las destrezas de listening, speaking, reading y writing. Con este alto porcentaje de acuerdo, se puede observar que efectivamente las estrategias metodológicas influyen significativamente en la formación académica de los estudiantes del cuarto semestre en la asignatura de inglés de la facultad de Psicología de la Universidad de Guayaquil.

\section{Conclusiones.}

La información y los resultados obtenidos mediante las encuestas a las autoridades docentes, padres de familia y estudiantes han proporcionado aportes significativos a la investigación. Ya que el objetivo principal es el de analizar la incidencia de las estrategias metodológicas en la formación 
académica de los estudiantes del cuarto semestre en la asignatura de inglés se detallan los resultados que se encontraron a lo largo del presente proyecto:

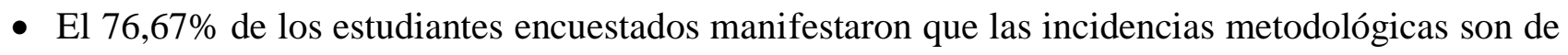
valiosa ayuda al momento de potenciar el proceso educativo.

- Los estudiantes están de acuerdo en que los Docentes del área de inglés deben capacitarse en cursos, seminarios, talleres, etc. sobre actualización de las estrategias metodológicas utilizadas en la actualidad.

- Los estudiantes manifestaron su interés en apoyar la aplicación de una nueva guía de estrategias metodológicas, con el fin de mejorar la relación enseñanza aprendizaje entre los estudiantes y los Docentes

Con respecto al diagnóstico de la formación académica actual de los estudiantes de la Facultad de Psicología de la Universidad de Guayaquil, se presentan los siguientes resultados:

- El $44.11 \%$ estudiantes se encuentran satisfechos con la formación académica que reciben actualmente en la Facultad de Psicología con respecto a la enseñanza del idioma inglés.

- El $43.7 \%$ se encuentra satisfecho con el programa de estudios de manera general que reciben.

Se debe indicar además que los estudiantes al ser motivados con las estrategias metodológicas usadas en el aula, se observó una participación más activa y pudieron desenvolverse de mejor manera en el transcurso de la clase.

La conclusión final es que las estrategias metodológicas influyen significativamente en la formación académica de los estudiantes del cuarto semestre de la asignatura de Inglés de la Facultad de Psicología de la Universidad de Guayaquil. 


\section{Recomendaciones.}

Las recomendaciones que se presentaron a lo largo de la investigación fueron las que se detallan a continuación:

- Los docentes deben estar en constante preparación e investigación sobre el manejo de estrategias activas para la enseñanza del inglés y de esta manera conseguir una mayor participación de los estudiantes.

- Fortalecer el conocimiento de los docentes de la Facultad al utilizar las estrategias metodológicas realizadas en esta investigación, con el fin de mejorar el proceso de interaprendizaje

- Concientizar a los estudiantes y enfatizar en la importancia que tiene el idioma Inglés en el mundo actual, recordando siempre que el idioma Inglés no es sólo una materia sino también una herramienta necesaria para el futuro profesional de los estudiantes.

- Las estrategias que utilizan los docentes para la enseñanza del idioma Inglés, deben seguir una estructura metodológica para alcanzar un mejor resultado.

- En el proceso de interaprendizaje del idioma Inglés, hoy en día se hace necesario el uso de recursos tecnológicos para mejorar los resultados, por lo que se recomienda la adquisición de nuevos programas informáticos que utilicen dichas estrategias.

\section{Bibliografía.}

Brandt, M. (1998). Estrategias de evaluación Barcelona. España

Cabrera, Geovanna (2010). La importancia del idioma inglés en la formación profesional. Pág.1

Ley Orgánica de Educación Superior (LOES). Articulo 124

Morales, M (2012). Implementación De Estrategias Metodológicas. Pág. 5

Zaldívar, Ricardo. Técnicas Activas Participativas como alternativa para el desarrollo de la Comunicación Oral. 1997. T OSOL The Oxford School of Language. 